\title{
Electroconvulsive Therapy for Neuropathic Pain: A Case Report and Literature ReView
}

\author{
Salahadin Abdi, MD, PhD, Arita Haruo, MD, and Joshua Bloomstone, MD
}

Objective: To describe a case of intractable brachial plexopathy-induced neuropathic pain syndrome treated with electroconvulsive therapy after a failed trial of conventional drugs and interventional pain management.

Case Report: A 32-year-old male had chronic intractable neuropathic pain of the right upper extremity and shoulder for about
10 years, due to brachial plexopathy. He tried multiple pain medications and underwent various interventional pain procedures without significant pain relief. When the patient subsequently developed severe depression with suicidal ideation, he underwent electroconvulsive therapy, which significantly improved the depression and pain for two months.

Discussion: There is a growing list of non- psychiatric conditions that may be treated with electroconvulsive therapy. Chronic intractable pain with or without depression has been on and off the list for years. Further studies may eventually demonstrate efficacy of ECT for intractable neuropathic pain syndromes.

Keywords: Electroconvulsive therapy, neuropathic pain, depression, intractable pain
Neuropathic pain is defined as pain that results from damage or abnormal function of the central or peripheral nervous system. Neuropathic pain can be difficult to manage using conventional treatment modalities. The usual treatments include opioids, anticonvulsants, antidepressants, antiarrhythmics and interventional procedures (e.g., spinal cord stimulators, nerve blocks, and neurosurgical ablation). Unfortunately, many patients fail these treatment modalities. Though not well studied, a few case reports illustrate the benefit of electroconvulsive therapy (ECT) in the management of chronic pain syndromes.

The exact origin of ECT is unknown. It probably dates back to the medical usage of eels by the Romans to treat headache and later the experiments of Italian naturalists with electricity (1). Further, the physician Galen employed electricity in the treatment of gout and other illnesses. Later, a Hungarian psychiatrist,

From Departments of Anesthesiology, Perioperative Medicine and Pain Management, Jackson Memorial Hospital, University of Miami School of Medicine

Miami, Florida and Massachusetts General Hospital, Harvard Medical School, Boston, Massachusetts. Address Correspondence: Salahadin Abdi, MD PhD, University of Miami School of Medicine, Miami, FL 33136.

E-mail: sabdi@med.miami.edu

Support:There was no external funding in preparation of this manuscript

Conflict of Interest: None
Laszlo Meduna, was credited with the first modern use of convulsive therapy. $\mathrm{He}$ was intrigued by the studies of Nyiro and Jablonski (2) in the 1920s, which demonstrated that patients with schizophrenia seemed to have a lower incidence of epilepsy than the general population. They postulated that dementia praecox (the old term for schizophrenia) had a curative effect on epilepsy, and Meduna wondered if the reverse might be true. He found that convulsions induced with intravenous Metrazol or intramuscular camphor could treat psychosis. Though Meduna is credited as the father of convulsive therapy, it was not until the late 1930s that Drs. Bini and Cerletti in Italy were successful in using electric currents to induce therapeutic seizures (3).

We describe a case of a 32-year-old man with a neuropathic pain syndrome who had received various treatments without satisfactory results. Subsequently, he was treated with electroconvulsive therapy for concomitant depression, which improved the depression and alleviated the chronic pain.

\section{Case Description}

Our patient was a 32-year old male with a 10-year history of chronic right arm and shoulder pain due to chronic idiopathic brachial neuritis. His pain started spontaneously as an aching, burning pain in the right shoulder, which then spread down the arm to the hand, and proximally to the lateral portion of the right side of the neck. He also complained of pain radiating into the scapular area and toward the thoracic spinal column. He described the pain as burning, sharp and crushing in nature. There was no particular activity that worsened the pain. Of note, in the early 1990s for a year or two, his pain nearly resolved spontaneously. Following a bout of prostatitis, the pain flared up again. Subsequently, the pain became progressively worse, to the level of 9-10/10 on the visual analog scale.

His past medical history included hypertension, seizure disorder and depression. He was treated as an inpatient and outpatient multiple times for depression. On physical examination, his right arm was noted to have decreased sensation to light touch and cold along the posterior aspect of the arm. There was hyperesthesia to pinprick in the right posterior shoulder area, right posterior arm, and the distal lateral wrist, encompassing an area of approximately $5 \mathrm{~cm}$ by $7 \mathrm{~cm}$. There was obvious atrophy of the pectoralis muscle. He was able to demonstrate at least 3/5-muscle strength in the shoulder.

MRI of his brachial plexus showed no anatomical abnormality, and MRI of the cervical spine showed no cord or nerve compression. An EMG showed chronic changes consistent with brachial 
plexopathy with involvement of the infraspinatus and supraspinatus muscles.

Treatment included continuous infusions of local anesthetics via an interpleural catheter, an intravenous lidocaine test, stellate ganglion blocks, TENS and interscalene brachial plexus blocks, all of which resulted in temporary mild pain relief, but no long-lasting relief. Medications included methadone $100 \mathrm{mg}$ a day in divided doses, hydromorphone $2 \mathrm{mg}$ orally every 8 hours as needed, gabapentin $1200 \mathrm{mg} 3$ times a day, clonidine 0.1 $\mathrm{mg}$ daily, clonazepam $1 \mathrm{mg}$ four times a day, and ibuprofen $800 \mathrm{mg} 3$ times a day.

Polypharmacy, interventional procedures, and behavioral pain management were unsatisfactory. He developed recurrent severe depression and attempted suicide. He was admitted to a psychiatric hospital for depression and suicidal ideation. While hospitalized, he underwent five right unilateral electroconvulsive treatments under methohexital ( 80 $\mathrm{mg}$ ) and succinylcholine (100 mg) anesthesia, with an average seizure duration of 62 seconds. He experienced relief of both the chronic pain and depression.

The patient spent approximately 2 weeks in the psychiatric hospital and then was discharged home. Subsequently, he did relatively well and began to taper his medications, including the opioids. During the recovery phase, he rated his pain as $3 / 10$ on a visual analog scale. He was happy with the response and increased his activity level significantly. Unfortunately, eight weeks later, the pain recurred. Although it is tempting to attribute the entire pain syndrome to a psychiatric disorder, this patient had bona fide evidence of brachial plexopathy and appeared to be in pain. The ECT treatments correlated with the 8-week period of pain relief.

\section{Discussion}

It is widely observed and acknowledged that there is an association between chronic pain and depression. Consequently, many physicians initially believed that antidepressants and psychotropic drugs might relieve chronic pain by improving depression. It is true that relatively small doses of tricyclic antidepressants can improve neuropathic pain, and adrenergic and/or serotonergic mechanisms are hypothesized.

Although ECT may be considered a nonpharmacologic method of treating depression, there are undoubtedly signifi- cant pharmacologic effects of ECT on the brain. ECT is often the only known effective treatment for severe depression resistant to medications. ECT may also be an effective and relatively safe treatment for psychosis and mania.

Not surprisingly, ECT is rarely considered for chronic intractable pain syndromes today, although there is an old, but rather sparse literature on the therapeutic use of ECT for pain. Mandel (4) reported that ECT alleviated symptoms for four of six patients suffering from chronic pain and depression. All of those patients had failed treatment with tricyclic antidepressants. Thus, he suggested that unilateral ECT might be the treatment of choice for patients suffering from chronic pain and intractable depression.

Bloomstein et al (5) reported that out of twenty-one patients who received ECT for concurrent affective symptoms, twenty experienced improvement in their pain levels, and concluded that ECT could be an effective treatment modality for patients who have chronic pain complicated by affective symptoms. Further, King et al (6) described a patient with depression who developed RSD and was helped by ECT. The patient had total resolution of pain for several hours after the first treatment. Over the course of the full ECT series, pain, vasculature changes of RSD, and depression resolved completely.

On the other hand, McCance et al (7) reported that ECT was not effective in the treatment of post-stroke thalamic pain. The authors performed a pilot study in 3 patients who received six bilateral ECT treatments over a period of two weeks and observed no improvement in thalamic pain or mood. Furthermore, Salmon et al (8) could not verify a positive response to unilateral ECT treatment for thalamic pain. They reported that unilateral ECT applied to four patients with intractable thalamic pain did not improve pain or affective profiles. The authors also measured venous plasma endorphins during the course of ECT therapy and found no significant correlations with ECT treatment. Interestingly, unilateral ECT in these patients was not effective for depression, but bilateral ECT was effective. They hypothesized that bilateral ECT resulted in current passage through the diencephalon, including the thalamus and hypothalamus $(9,10)$. These structures are well known pathways for pain sensation and perception.

Even though there is little definitive evidence that ECT is effective for chronic pain, the idea is intriguing. Indeed, it has been suggested that some complaints of chronic pain represent a symptom of underlying depression $(4,10,11)$. Lason et al (12) suggested that the transient improvement in pain observed in four out of ten patients after ECT might be related to temporary ECT-induced delirium. Indeed, changes in a number of neurotransmitter systems may occur with ECT. The persistence of EEG slow wave activity following ECT and its correlation with clinical improvement suggests that a physical alteration in cerebral activity has occurred. Both antidepressants and ECT seem to alter serotonergic neurotransmission. ECT up-regulates 5-HT2, and antidepressants down-regulate receptor expression (13).

Interestingly, Lason et al (12) observed a differential effect of ECT on the prodynorphin and pro-opiomelanocortin system in the rat. They studied the effect of single and repeated ECT on immunoreactive (ir) dynorphin and betaendorphin levels in the rat brain, spinal cord and pituitary. They reported that there was a reduction in ir-dynorphin and beta-endorphin levels in the hypothalamus and beta-endorphin in the pituitary, when ECT was applied as a single dose. However, repetitive applications resulted in significant increases of ir-dynorphin, but not beta-endorphin in the hypothalamus. They concluded that the observed prolonged changes in dynorphin levels after chronic ECT may play a role in enhanced post-shock opiate-like behavior, as well as provide a mechanism for ECT therapy. Furthermore, Hong et al (14) and Sarne et al (15) have reported that ECT led to a long-lasting increase in met- and leu-enkephalin levels in the hypothalamus as well as in the rat limbic system.

Even though ECT was used for various types of intractable chronic pain during the 1940s and 1950s, it is not now often used for pain, for obvious reasons. There are no controlled studies to clarify the role of ECT for intractable pain and there are only a few case reports with variable results. The negative image associated with ECT makes it unlikely that controlled trials will ever be done. Despite the fact that the risk of prolonged amnesia 
or induced psychosis from ECT is well under $1 \%$ and the estimated mortality rate following ECT is less than $0.03 \%$ (16). More recent reports confirm the safety of ECT, even for elderly patients (17). Nonetheless, concerns about such factors as amnesia and injury from ECT make the option rather unpopular, despite the fact that more recent evidence, again from case reports, suggests that ECT may benefit subsets of patients with chronic pain. Recently, ECT has been reported to help patients with phantom limb pain (18), headache (19), and CRPS (20).

In conclusion, although ECT is widely accepted as a treatment for severe depression, it is seldom used in the treatment of chronic pain, despite the fact that chronic pain and depression frequently occur together. Our report is intended to rekindle a discussion about the possible use of ECT in depressed patients presenting with intractable pain syndromes. Although prospective randomized studies are needed to demonstrate the effectiveness of ECT for the treatment of chronic pain, such studies most likely will never be done. Nonetheless, there is anecdotal evidence that ECT may alleviate symptoms of depression and pain in some patients who have failed conventional treatment.

\footnotetext{
Author Affiliation:

Salahadin Abdi, MD PhD

Professor and Chief

Division of Pain Medicine

University of Miami Pain Center

Dept. of Anesthesiology, Periopera-

tive Medicine and Pain Management

JMH/University of Miami School of

Medicine

Miami, FL 33136

E-mail: sabdi@med.miami.edu

Arita Haruo, MD

Department of Anesthesiology and

Critical Care

The Massachusetts General Hospital

Harvard Medical School

Boston, MA 02114

Joshua Bloomstone, MD

Department of Anesthesiology and

Critical Care

The Massachusetts General Hospital Harvard Medical School

Boston, MA 02114
}

\section{References}

1. Harms E. The origin and early history of electrotherapy and electroshock. Am J Psychiatry 1956; 111:933-934.

2. Nyiro J, Jablonsky A. Einige daten zur prognose der Epilepsie, mit besonderer rucksicht auf die konstitution. Psychiatr Neurol Wochenschr 1929; 31:547-549.

3. Cerletti U, Bini L. Unnuevo metodo di shockterapie “L'elettroshock.” Bollettino Accademia Medica Roma 1938; 64:136138.

4. Mandel MR. Electroconvulsive therapy for chronic pain associated with depression. Am J Psychiatry 1975; 132:632-636.

5. Bloomstein JR, Rummans TA, Maruta T et al. The use of electroconvulsive therapy in pain patients. Psychosomatics 1996; 37: 374-379.

6. King JH, Nuss S. Reflex sympathetic dystrophy treated by electroconvulsive therapy: Intractable pain, depression, and bilateral electrode ECT. Pain 1993; 55:393396.

7. McCance $\mathrm{S}$, Hawton $\mathrm{K}$, Brighouse $\mathrm{D}$ et al. Does electroconvulsive therapy (ECT) have any role in the management of intractable thalamic pain? Pain 1996; 68: 129-131.

8. Salmon JB, Hanna MH, Williams $M$ et al. Thalamic pain-the effect of electroconvulsive therapy (ECT). Pain 1988; 33:67-71.

9. Abrams R, Taylor MA. Diencephalic stimulation and the effects ECT in endogenous depression. Br J Psychiat 1976; 129:482485 .

10. Bradley JJ. Severe localized pain associated with the depressive syndrome. BrJPsy- chiat 1963; 109:741-745.

11. Weiss DM. The use of electro-shock therapy for atypical and intractable pain. Trans Am Neurol Ass 1957; 82:166-168.

12. Lason W, Przewlocka B, Przewlocki R. Single and repeated electroconvulsive shock differentially affects the prodynorphin and pro-opiomelanocortin system in the rat. Brain Res 1987; 403:301-307.

13. Weiner SJ, Ward TN, Ravaris CL. Headache and electroconvulsive therapy. Headache 1994; 34:155-159.

14. Hong JS, Gillin JC, Yang HY et al. Repeated electroconvulsive shocks and the brain content of endorphins. Brain Res 1979; 177:273-278.

15. Sarne Y, Weissman BA, Urca G. Differential effects of long-term electroconvulsive shock on brain levels of enkephalin and humoral-endorphin. J Neurochem 1982; 39:1478-1480.

16. Spencer J. Psychiatry and convulsant therapy. Med J Aust 1977; 1:844-847.

17. van der Wurff FB, Stek ML, Hoogendijk WJ et al. The efficacy and safety of ECT in depressed older adults: a literature review. Int J Geriatr Psychiatry 2003; 18:894-904.

18. Rasmussen KG, Rummans TA. Electroconvulsive therapy for phantom limb pain. Pain 2000; 85:297-299.

19. Rasmussen KG, Rummans TA. Electroconvulsive therapy in the management of chronic pain. Curr Pain Headache Rep 2002; 6:17-22.

20. McDaniel WW. Electroconvulsive therapy in complex regional pain syndromes. J ECT 2003; 19:226-229. 
